\title{
The Collembola of North Forests of Iran, List of Genera and Species
}

\author{
Masoumeh Shayanmehr ${ }^{1}$ and Elliyeh Yahyapour ${ }^{2}$ \\ Department of Plant Protection, Faculty of Crop Sciences, Sari University of Agricultural Sciences and Natural Resources, Sari, \\ Mazandaran 582, Iran \\ Department of Entomology, Faculty of Agricultural Sciences, Islamic Azad University, Arak-Branch, Arak 38135/567, Iran
}

\begin{abstract}
The Collembola fauna of Iran has received little attention and this applies in particular to the Hyrcanian forests in northern Iran. In this study, the list of Collembola from north forests of Iran, and collected information such as study site, until March 2019 are listed. At present, 107 species, belonging to 14 families and 51 genera are known from northern forests of Iran.
\end{abstract}

Key words: Collembola, checklist, forest, Iran.

\section{Introduction}

Hyrcanian forests are located in northern Iran and mostly are composed of deciduous trees. The climate of south Caspian region is humid with most precipitation occurring in autumn, winter and spring. Soil and leaf litter in these forests are occupied by different soil-dwelling animals especially Collembola [1]. The Collembola, commonly called "spring-tails", is small, entognathous, wingless hexapods possessing a spring-like forked jumping organ, the furcula, underneath the fourth abdominal segment. There are about 9000 described species of Collembola worldwide [2]. The first Iranian species of Collembola is from wheat and alfalfa fields in Khuzestan [3]. Cox who travelled to Northern Provinces in Iran, collected and identified 70 species of 30 genera and five families [4]. During last decade, many researchers worked on Collembola fauna of Iran [5] and provided a list of their reported genera and species from Iran up to 2013. Amongst these species, there are several reported species from forests. High abundant of Collembola in forest soil gets researchers' attention to

Corresponding author: Eliye Yahyapour, Ph.D., research field: entomology. work on their fauna [6-22]. Here, authors provide an update to the list of Collembola from northern forests of Iran published from 2013 to 2019. Obviously, the fauna of forests of Iran is unknown, this present study aims at contributing to close this gap of knowledge, concentrating the unique Hyrcanian forests and providing information on the fauna of Collembola in different soil layers and their seasonal variation.

\section{Materials}

A search of the literature was undertaken for any published reference to any member of the Collembola in forests of Mazandaran in north Iran. In almost all cases, authors were able to access an electronic version of the published work (in pdf format). Data on species were abstracted from these sources then maintained and managed in a Word file in preparation for publication. The samples were placed in dark polythene bags. Collembola was extracted from soil and leaf litter by Berlese funnels. Animals were collected in water and separated under a dissecting microscope. Some specimens were cleared in Nesbit's solution, then were mounted on Hoyer's medium to make microscopic slides. Then they were identified as genera and species levels using valid keys. 


\section{Results and Discussion}

The checklist of genera and species of Collembola of Mazandaran forests in north of Iran presented here includes a total of 107 species and 51 genera belonging to 14 families. The most diverse families are Entomobryidae, Isotomidae and Onychiuridae with 24, 22 and 14 reported species, respectively. The less diverse families are Oncopoduridae, Sminthurididae, Tullbergiidae and Arrhopalitidae with $1,1,2$ and 2 reported species, respectively.

\section{Table 1 Checklist of Collembola from the Northern forest of Iran.}

\begin{tabular}{|c|c|c|}
\hline Taxonomy/order/family/genera/species & References & Habitat \\
\hline \multicolumn{3}{|l|}{ Poduromorpha (Börner, 1906) } \\
\hline \multicolumn{3}{|l|}{ Family Neanuridae (Börner, 1901) } \\
\hline \multicolumn{3}{|l|}{ Genus Pseudachorutes (Tullberg, 1871) } \\
\hline Pseudachorutes corticolus (Schaffer, 1897) & Sari, Semeskandeh [18] & Forest, leaf litter \\
\hline Pseudachorutes parvulus (Börner, 1901) & $\begin{array}{l}\text { Sari, Shahid Zare forest, Chahardangeh, Langar } \\
\text { village; Dasht-e-Naz [20] }\end{array}$ & Soil and leaf litter \\
\hline Pseudachorutes subcrassus (Tullberg, 1871) & Sari, Semeskandeh [18] & Forest, soil and leaf litter \\
\hline \multicolumn{3}{|l|}{ Subfamily Neanurinae } \\
\hline \multicolumn{3}{|l|}{ Genus Bilobella (Caroli, 1912) } \\
\hline Bilobella aurantiaca (Caroli, 1912) & Mazandaran [5] & Soil, leaf litter \\
\hline \multicolumn{3}{|l|}{ Genus Endonura (Cassagnau, 1979) } \\
\hline Endonura longirostris (Smolis et al., 2017) & Behshahr, Abbas-Abad forest; Noor, Kadirsar [15] & $\begin{array}{l}\text { leaf litter and soil rotting } \\
\text { wood }\end{array}$ \\
\hline Endonura paracentaurea (Smolis et al., 2017) & Behshahr, Abbas-Abad forest [15] & Soil, leaf litter \\
\hline \multicolumn{3}{|l|}{ Genus Deutonura (Cassagnau, 1979) } \\
\hline Deutonura decolorata (Gama and Gisin, 1964) & Mazandaran [5] & Soil, leaf litter \\
\hline Deutonura persica (Smolis et al., 2018) & Neka, Hezar Jarib forest [16] & $\begin{array}{l}\text { Dead wood, moss on tree, } \\
\text { leaf litter, dead wood in a } \\
\text { hole of Persian ironwood }\end{array}$ \\
\hline \multicolumn{3}{|l|}{ Genus Neanura (MacGillivray, 1893) } \\
\hline Neanura deharvengi (Smolis et al., 2018) & Behshahr, Abbas-Abad forest; Noor, Kadirsar [16] & Leaf litter and soil \\
\hline Neanura muscorum (Templeton, 1835) & Sari [5] & Leaf litter \\
\hline \multicolumn{3}{|l|}{ Genus Persanura (Mayvan et al., 2015) } \\
\hline Persanura hyrcanica (Mayvan et al., 2015) & Sari, Semeskandeh $[13,18]$ & $\begin{array}{l}\text { Soil and litter, } \\
\text { oak-hornbeam }\end{array}$ \\
\hline \multicolumn{3}{|l|}{ Family Hypogastrurida (Börner,1906) } \\
\hline \multicolumn{3}{|l|}{ Genus Ceratophysella (Börner, 1932) } \\
\hline Ceratophysella denticulata (Bagnall, 1941) & Sari; Noor; Savadkooh; Babol $[5,7,8,10,17]$ & Diva, leaf litter \\
\hline Ceratophysella stercoraria (Stach, 1963) & $\begin{array}{l}\text { Babol; Sari, Chahardangeh, Langar village, Salar } \\
\text { Darreh forest; Fereydunkenar; Savadkooh; } \\
\text { Qaemshahr; Neka; Behshahr; Amol, Razakeh } \\
\text { forest, Halumsar forest; Babolsar; Tonekabon, Soil } \\
{[7,10,12,18,20,23]}\end{array}$ & Soil, leaf litter \\
\hline \multicolumn{3}{|l|}{ Genus Hypogastrura (Bourlet, 1839) } \\
\hline Hypogastrura manubrialis (Tullberg, 1869) & Amol [5] & Soil, leaf litter \\
\hline $\begin{array}{l}\text { Hypogastrura martiani (Skarżyński and Kaprus', } \\
\text { 2009) }\end{array}$ & Babol, Shiadeh forest [23] & Oak forest \\
\hline Hypogastrura purpurescens (Lubbock, 1867) & Fereydunkenar $[6,10]$ & $\begin{array}{l}\text { Soil and leaf litter, oak } \\
\text { forest }\end{array}$ \\
\hline
\end{tabular}


Table 1 to be continued

\begin{tabular}{|c|c|c|}
\hline Hypogastrura tullbergi (Schäffer, 1900) & Mazandaran [5] & Soil, leaf litter \\
\hline \multicolumn{3}{|l|}{ Genus Schoetella (Schäffer, 1896) } \\
\hline Schoetella ununguiculata (Tullberg, 1869) & $\begin{array}{l}\text { Larijan; Savadkooh; Babol, Shiadeh forest, } \\
\text { Bezchaft forest }[7,10,11,23]\end{array}$ & Soil, leaf litter \\
\hline \multicolumn{3}{|l|}{ Genus Triacanthella (Schäffer, 1897) } \\
\hline $\begin{array}{l}\text { Triacanthella intermedia } \\
\text { (Dunger and Zivadinovic, 1984) }\end{array}$ & Juybar [8] & Leaf litter \\
\hline \multicolumn{3}{|l|}{ Genus Xenylla (Tullberg, 1869) } \\
\hline Xenylla maritima (Tullberg, 1869) & $\begin{array}{l}\text { Neka; Savadkooh; Amol, Halumsar forest; Babol, } \\
\text { Bzchaft forest }[7,10,23]\end{array}$ & $\begin{array}{l}\text { Soil, leaf litter, moss on } \\
\text { tree }\end{array}$ \\
\hline Xenylla mediterranea (da Gama, 1964) & $\begin{array}{l}\text { Amol, Halumsar Forest Park; Babol, Bezchaft } \\
\text { forest park [23] }\end{array}$ & Moss on tree and rock \\
\hline Xenylla welchi (Folsom, 1916) & Sari $[5]$ & Leaf litter \\
\hline \multicolumn{3}{|l|}{ Family Onychiuridae (Lubbok, 1867) } \\
\hline \multicolumn{3}{|l|}{ Subfamily Onychiurinae } \\
\hline \multicolumn{3}{|l|}{ Genus Heteraphorura (Bagnall, 1948) } \\
\hline Heteraphorura iranica (Kaprus' et al., 2017) & $\begin{array}{l}\text { Nowshahr, Kajour, Lashkenar; Babolsar, Bahnamir; } \\
\text { Sari, Semeskandeh forest [19] }\end{array}$ & Leaf litter \\
\hline Heteraphorura japonica (Yosii, 1967) & Sari, Semeskandeh forest; Juybar $[12,18]$ & Leaf litter \\
\hline \multicolumn{3}{|l|}{ Genus Hymenaphorura (Gervais, 1841) } \\
\hline Hymenaphorura sibirica (Tullberg, 1876) & Mazandaran [5] & Soil \\
\hline \multicolumn{3}{|l|}{ Genus Onychiuroides (Bagnall, 1948) } \\
\hline Onychiuroides granulosus (Stach, 1930) & Mazandaran [5] & Soil \\
\hline Onychiuroides pseudogranulosus (Gisin, 1951) & Mazandaran [5] & Soil \\
\hline \multicolumn{3}{|l|}{ Genus Orthonychiurus (Stach, 1954) } \\
\hline Orthonychiurus folsomi (Schäffer, 1900) & Sari $[5]$ & $\begin{array}{l}\text { Leaf litter, soil and leaf } \\
\text { litter under pine and oak }\end{array}$ \\
\hline Orthonychiurus rectopapillatus (Stach, 1933) & Mazandaran [5] & Soil \\
\hline \multicolumn{3}{|l|}{ Genus Protaphorura (Absolon, 1901) } \\
\hline Protaphorura bicampata (Gisin, 1956) & Mazandaran (Shayanmehr et al., 2013) & Soil \\
\hline Protaphorura fimata (Gisin, 1952) & Larijan (Shayanmehr et al., 2013; Balvasi et al., 2015) & Soil, leaf litter \\
\hline Protaphorura ombrophila (Stach, 1960) & Sari, Semeskandeh forest (Ghasemi Cherati, 2017) & Soil \\
\hline Protaphorura quadriocellata (Gisin, 1947) & Mazandaran (Shayanmehr et al., 2013) & Soil \\
\hline Protaphorura sakatoi (Yosii, 1966) & $\begin{array}{l}\text { Noor, Sisangan wildlife refuge; Nowshahr, Kojur, } \\
\text { Lashkenar; Babol, Khoshrudpay; Sari, } \\
\text { Semeskandeh forest; Juybar (Yoosefi Lafooraki, } \\
\text { 2014; Yoosefi Lafooraki and Shayanmehr, 2015a; } \\
\text { Alijani-Ardeshir } \text { et al., 2017; Ghasemi Cherati, 2017) }\end{array}$ & $\begin{array}{l}\text { Soil and leaf litter under } \\
\text { elm tree }\end{array}$ \\
\hline \multicolumn{3}{|l|}{ Genus Thalassaphorura (Bagnall, 1949) } \\
\hline Thalassaphorura encarpata (Denis, 1931) & $\begin{array}{l}\text { Sari; Mahmood-abad; Noor; Babolsar; Qaemshahr } \\
\text { (Yoosefi Lafooraki, 2014; Yoosefi Lafooraki and } \\
\text { Shayanmehr, 2013, 2015a) }\end{array}$ & Soil under oak trees \\
\hline \multicolumn{3}{|l|}{ Family Tullbergiidae (Bangel, 1935) } \\
\hline \multicolumn{3}{|l|}{ Genus Mesaphorura (Börner, 1901) } \\
\hline Mesaphorura krausbaueri (Börner, 1901) & Mazandaran (Shayanmehr et al., 2013) & Soil \\
\hline \multicolumn{3}{|l|}{ Genus Tullbergia (Lubbock, 1876) } \\
\hline Tullbergia simplex (Gisin, 1958) & $\begin{array}{l}\text { Savadkooh (Yoosefi Lafooraki and Shayanmehr, } \\
\text { 2013; Yoosefi Lafooraki, 2014) }\end{array}$ & Soil \\
\hline
\end{tabular}


Table 1 to be continued

\section{Order Entomobryomorpha \\ Family Oncopoduridae \\ (Carl and Lebedinsky, 1905) \\ Genus Oncopodura (Carl and Lebedinsky, 1905) \\ Oncopodura ambigua (Christiansen, 1957) \\ Family Tomoceridae (Schäffer, 1896) \\ Genus Pogonognathellus (Tullberg, 1871)** \\ Pogonognathellus flavescens (Tullberg, 1871)**}

Genus Tomocerus (Nocolet, 1842)

Tomocerus minor (Lubbock, 1862)

Tomocerus vulgaris (Tullberg, 1871)

Family Isotomidae (Schäffer, 1896)

Genus Ballistura (Börner, 1906)

Ballistura schoetti (Dalla Torre, 1895)

Genus Hemisotoma (Bagnall, 1949)

Hemisotoma thermophila (Axelson, 1900)

Hemisotoma orientalis (Stach, 1947)

Hemisotoma pontica (Stach, 1947)

Genus Folsomia (Willem, 1902)

Folsomia brevifurca (Bagnall, 1949)

Folsomia candida (Willem, 1902)

Folsomia fimetaria (Linnaeus, 1758)

Folsomia inoculata (Stach, 1946)**

Folsomia ksenemani (Stach, 1947)

Folsomia penicula (Bagnall, 1939)

Folsomia quadrioculata (Tullberg, 1871)

Genus Folsomides (Stach, 1922)

Folsomides parvulus (Stach, 1922)

Genus Isotoma (Bourlet, 1839)

Isotoma viridis (Bourlet, 1839)

Genus Isotomiella (Bagnall, 1939)

Isotomiella minor (Schäffer, 1896)

Genus Isotomurus (Börner, 1903)

Isotomurus afghanicus (Yosii, 1963)
Sari (Shayanmehr et al., 2013)

Soil, leaf litter

Sari, Chahardangeh, Langar village; Tonekabon; Babol, Filband; Neka, Hezar Jarib forest [21]

Soil and leaf litter

Mazandaran [5]

Soil

Savadkooh, Forest of Jawarom wildlife refuge; Larijan; Sari, Semeskandeh forest $[5,7,11,12,18]$

Soil, leaf litter

Neka [20]

Soil, leaf litter

Soil (Ulmus sp., Ficus sp., Ziziphus sp., Populus sp.) leaf litter, soil and leaf litter under poplar and oak Soil, leaf litter

Mazandaran [5]

Sari; Simorq; Mahmood-abad; Qaemshahr [5-7]

Mazandaran [5]

Soil

Sari [5]

Mazandaran [5]

Sari, Dasht-e-Naz forest, Shahid Zare forest [22]

Noor, Royan, Kodirsar; Savadkooh, Alasht, Serin village; Forest of Jawarom wildlife refuge $[6,7]$

Nowshahr, Kojur, Lashkenar; Bahnamir; Sari,

Chahardangeh, Langar village, Semeskandeh forest $[5-7,12,18,20]$

Mazandaran [5]

Sari, Dasht-e-Naz forest; Savadkooh, Forest of Jawarom wildlife refuge; Alasht, Serin village; Babolsar; Qaemshahr [5, 7, 20]

Soil (Morus sp.), leaf litter, soil of black cherry garden

Sari; Babol, Bandpay Sharghi [5, 17]

Soil, leaf litter

Sari, Semeskandeh forest; Nowshahr; Noor, Forest of Sisangan wildlife refuge [5-7, 12, 18]

Soil (Quercus sp., Prunus sp., Pinus sp.), leaf litter, soil of lawn, soil next to river

Mahmood-abad; Miandorud; Noor, Forest of Sisangan, wildlife refuge; Amol; Neka, Hezarjerib Soil leaf litter forest $[6,7]$ 
Table 1 to be continued

Isotomurus italicus (Carapelli et al., 1995)

Isotomurus palustris (Muller, 1776)

Genus Parisotoma (Bagnall, 1940)

Parisotoma notabilis (Schäffer, 1896)

Genus Proisotoma (Börner, 1901)

Proisotoma minima (Absolon, 1901)

Proisotoma minuta (Tullberg, 1871)

Proisotoma subminuta (Denis, 1931)

Genus Pseudisotoma (Handschin, 1924)

Pseudisotoma sensibilis (Tullberg, 1876)

Family Entomobryidae (Schäffer, 1896)

Subfamily Entomobryinae

Genus Entomobrya (Rondani, 1861)

Entomobrya obscuralla (Brown, 1926)

Entomobrya atrocincta (Schött, 1986)

Entomobrya corticalis (Nicolet, 1841)*

Entomobrya dollfusi (Denis, 1924)

Entomobrya lindbergi (Stach, 1960)

Entomobrya lanuginosa (Nicolet, 1841)

Entomobrya multifasciata (Tullberg, 1871)

Entomobrya nigrocincta (Denis, 1923)

Entomobrya schoetti (Stach, 1922)

Genus Sinella (Brook, 1882)

Sinella curviseta (Brook, 1882)

Sinella tenebricosa (Folsom, 1902)

Subfamily Lepidocyrtinae

Genus Lepidocyrtus (Bourlet, 1839)

Lepidocyrtus cyaneus (Tullberg, 1871)

Lepidocyrtus lanuginosus (Gmelin, 1788)

Lepidocyrtus ruber (Schött, 1902)

Genus Pseudosinella (Schaefer, 1897)

Pseudosinella imparipunctata

Pseudosinella octopunctata (Böner,1901)

Subfamily Seirinae

Genus Seira (Lubbock, 1870)

Seira domestica (Nicolet, 1842)
Sari, Semeskandeh forest $[12,18]$

Sari [5]

Noor, Royan, Kodirsar; Sari, Shahid Zare forest, $[5-7,20]$

Sari; Qaemshahr [5-7]

Mahmood-abad; Larijan [5-7, 11]

Amol [6, 7]

Noor, Royan $[6,7]$

Dead wood

Leaf litter

Sari, Semeskandeh forest, Dasht-e-Naz forest; Savadkooh $[5,18,22]$

Sari, Shahid Zare forest [21]

Sari [5]

Sari $[6,7]$

Mazandaran [5]

Leaf litter

Soil and leaf litter

Leaf litter

Soil (Alnus sp. and Ulmus sp.), Soil and leaf litter under walnut trees

Soil

Sari, Semeskandeh forest; Galugah; Juybar [5, 6] [18] Leaf litter

Sari, Chahardangeh, Langar village, Dasht-e-Naz, Salar Darreh forest [21]

Neka, Hezar Jarib forest [21]

Soil

Leaf litter

Savadkooh, Forest of Jawarom wildlife refuge; Qaemshahr [5, 6]

Babol, Ganjkola [17]

Soil, leaf litter under poplar

Leaf litter and soil

Mazandaran [5] Soil

Mazandaran [5] Soil

Mazandaran [5] Soil

Mazandaran [5] Soil

Sari, Semeskandeh forest; Babolsar; Larijan [5-8, Soil, leaf litter, moss on $11,18]$ rocks

Sari, Salar Darreh forest; Qaemshahr, Jadeh-Nezami,

Semeskandeh forest; Neka,

Leaf litter, soil (Magnolia sp., Ficus sp., Hezar Jarib forest; Babol, Filband [5, 18, 21]
Ulmus sp., Prunus sp.) 
Table 1 to be continued

\begin{tabular}{|c|c|c|}
\hline \multicolumn{3}{|l|}{ Subfamily Orchesellinae } \\
\hline \multicolumn{3}{|l|}{ Genus Heteromurus (Wankel, 1860) } \\
\hline Heteromurus gigans (Mari Muttt and Stomp, 1980) & $\begin{array}{l}\text { Sari, Semeskandeh forest, Dasht-e-Naz forest, } \\
\text { Shahid Zare forest; Babol, Filband [18, 22] }\end{array}$ & Leaf litter \\
\hline Heteromurus major (Moniez, 1889) & $\begin{array}{l}\text { Sari, Salar Darreh forest, Dasht-e-Naz, Shahid Zare } \\
\text { forest, Semeskandeh forest; Savadkooh, Lafoor; } \\
\text { Noor, Katel-shani, Noor, Forest of Sisangan } \\
\text { wildlife refuge; Larijan; Qaemshahr, Jadeh-Nezami; } \\
\text { Neka, Hezar Jrib forest; Babol, Filband [5, 6, 8, 12, } \\
\text { 18, 21] }\end{array}$ & Soil, leaf litter \\
\hline Heteromurus nitidus (Templeton, 1835) & $\begin{array}{l}\text { Savadkooh; Sari, Semeskandeh forest; Babol, } \\
\text { Ganjkola village, Diva village, Babolkenar, } \\
\text { Derazkola village }[5,6,8,12,18]\end{array}$ & Soil, leaf litter \\
\hline Heteromurus sexoculatus (Brown, 1926) & Mazandaran [5] & Soil \\
\hline Heteromurus variabilis (Martynova, 1974) & Sari, Semeskandeh forest [18] & Leaf litter \\
\hline \multicolumn{3}{|l|}{ Genus Orchesella (Templeton, 1836) } \\
\hline Orchesella cincta (Linnaeus, 1758) & $\begin{array}{l}\text { Savadkooh, Forest of Jawarom wildlife refuge; } \\
\text { Alasht, Serin village; Larijan; Babol, Filband; } \\
\text { Neka, Hezar Jarib forest }[6,8,11,21]\end{array}$ & $\begin{array}{l}\text { Leaf litter, moss on the } \\
\text { rock }\end{array}$ \\
\hline Orchesella spectabilis (Stach, 1960) & Sari, Semeskandeh forest $[18]$ & Leaf litter \\
\hline \multicolumn{3}{|l|}{ Order Neelipleona (Massoud, 1971) } \\
\hline \multicolumn{3}{|l|}{ Family Neelidae (Folsom, 1896) } \\
\hline \multicolumn{3}{|l|}{ Genus Megalothorax (Willem, 1900) } \\
\hline Megalothorax incertus (Börner, 1903) & Savadkooh $[6,7]$ & Soil, leaf litter \\
\hline $\begin{array}{l}\text { Megalothorax willemi (Schneider and D'Haese, } \\
\text { 2013) }\end{array}$ & $\begin{array}{l}\text { Neka; Behshahr; Noo; Sari, Semeskandeh forest }[6 \text {, } \\
7,18]\end{array}$ & Soil, leaf litter \\
\hline $\begin{array}{l}\text { Megalothorax perspicillum (Schneider and } \\
\text { D'Haese, 2013) }\end{array}$ & $\begin{array}{l}\text { Neka, Hezar Jrib forest; Behshahr, Abbas-Abad } \\
\text { forest }[6,7]\end{array}$ & Soil, leaf litter, dead wood \\
\hline \multicolumn{3}{|l|}{ Genus Neelus (Folsom, 1896) } \\
\hline Neelus murinus (Folsom, 1896) & Sari, Semeskandeh forest $[5,18]$ & \\
\hline \multicolumn{3}{|l|}{ Order Symphypleona (Börner, 1901) } \\
\hline \multicolumn{3}{|l|}{ Family Sminthurididae (Börner, 1906) } \\
\hline \multicolumn{3}{|l|}{ Genus Sphaeridia (Linnaniemi, 1912) } \\
\hline Sphaeridia pumilis (Krausbauer, 1898) & $\begin{array}{l}\text { Neka, Hezar jrib forest; Sari; Behshahr, } \\
\text { Abbas-Abad forest; Noor, Royan }[5,6,9]\end{array}$ & \\
\hline \multicolumn{3}{|l|}{ Family Katiannidae (Börner, 1913) } \\
\hline \multicolumn{3}{|l|}{ Genus Sminthurinus (Börner, 1901) } \\
\hline Sminthurinus aureus (Lubbock, 1862) & Sari [5] & Leaf litter \\
\hline Sminthurinus elegans (Fitch, 1863) & $\begin{array}{l}\text { Sari, Semeskandeh forest; Noor, Royan; Neka, } \\
\text { Hezar jrib forest; Behshahr, Abbas-Abad forest; } \\
\text { Juybar }[5,6,12,9,18]\end{array}$ & $\begin{array}{l}\text { Soil, leaf litter moss on } \\
\text { tree }\end{array}$ \\
\hline Sminthurinus gisini (da Gama, 1956) & Neka, Hezar jrib forest; Savadkooh, Alasht $[6,9]$ & Split of tree, moss on tree \\
\hline \multicolumn{3}{|l|}{ Family Arrhopalitidae (Stach, 1956) } \\
\hline \multicolumn{3}{|l|}{ Genus Arrhopalites (Börner, 1906) } \\
\hline Arrhopalites caecus (Tullberg, 1871)* & Tonekaon, Sari, Chahardangeh, Langar village [20] & Soil, leaf litter \\
\hline Arrhopalites principalis (Stach, 1945)** & Sari, Chahardangeh, Langar village [20] & Soil, leaf litter \\
\hline \multicolumn{3}{|l|}{ Family Sminthuridae (Lubbok, 1862) } \\
\hline Genus Allacma (Börner, 1906) & & \\
\hline
\end{tabular}


Table 1 to be continued

\begin{tabular}{|c|c|c|}
\hline Allacma fusca (Linnaeus, 1758) & Sari, Zare forest [24] & Leaf litter \\
\hline \multicolumn{3}{|l|}{ Genus Lipothrix (Börner, 1906) } \\
\hline Lipothrix lubbocki (Tullberg, 1872) & Sari, Semeskandeh forest; Tonekabon $[12,20]$ & Leaf litter \\
\hline \multicolumn{3}{|l|}{ Genus Paralipothrix (Bretfeld, 1999) } \\
\hline Paralipothrix natalicius (Ellis, 1974) & Sari, Semeskandeh forest [12] & Leaf Litter \\
\hline \multicolumn{3}{|l|}{ Genus Sminthurus (Latreille, 1804) } \\
\hline Sminthurus ghilarovi (Stebaeva, 1966) & Sari, Semeskandeh forest [12] & Leaf litter \\
\hline \multicolumn{3}{|l|}{ Genus Caprainea (Dallai 1970) } \\
\hline Caprainea marginata (Schott, 1893) & Sari, Semeskandeh forest [12] & Soil, leaf litter \\
\hline \multicolumn{3}{|l|}{ Family Dicyrtomidae (Börner, 1906) } \\
\hline \multicolumn{3}{|l|}{ Genus Dicyrtomina (Börner, 1903) } \\
\hline Dicyrtomina ornata (Nicolet, 1842) & Sari [5] & Leaf litter \\
\hline \multicolumn{3}{|l|}{ Genus Dicyrtoma (Bourlet, 1841) } \\
\hline Dicyrtoma ghilarovi (Bretfeld, 1996) & Sari, Semeskandeh forest [12] & Leaf litter \\
\hline Dicyrtoma minuta (Fabricius, 1783) & Mazandaran [5] & Soil \\
\hline Dicyrtoma fusca (Lubbock, 1873) & Sari [5] & Leaf litter \\
\hline
\end{tabular}

** New for Iranian fauna, *New for Mazandaran Province.

\section{Conclusions}

In this study, the list of Collembola from North Forests of Iran and collected information such as study site, habitats, e.g. soils, leaf litter, vegetation, and collectors have been presented between years 2013-3018. Moreover some samples were collected and identified by the author during 2016-2018. An up-to-date systematic checklist of Collembolans has been provided.

\section{References}

[1] Siadati, S., Moradi, H., Attar, F., Etemad, V., Hamzeh'ee, B., and Naqinezhad, A. 2010. "Botanical Diversity of Hyrcanian Forests: A Case Study of a Transect in the Kheyrud Protected Lowland Mountain Forests in Northern Iran." Phytotaxa 7: 1-18.

[2] Bellinger, P. F., Christiansen, K. A., and Janssens, F. 1996-2019. Checklist of the Collembola of the World. Available from: http://www.Collembola.org/taxa.

[3] Farrahbakhsh, G. 1961. A Checklist of Economically Important Insect and Other Enemies of Plant and Agricultural Products in Iran, Vol. 1. Department of Plant Protection, Ministry of Agriculture, Tehran, Iran, 153. (in press)

[4] Cox, P. 1982. "The Collembola Fauna of North and North Western Iran.” Entomologist's Monthly Magazine
118: $39-43$

[5] Shayanmehr, M., Yahyapour, E., Kahrarian, M., and Yoosefi Lafooraki, E. 2013. "An Introduction to Iranian Collembola (Hexapoda): An Update to the Species list." Zookeys 335: 69-83.

[6] Yoosefi Lafooraki, E. 2014. "Morphological ad Biological Identification of Collembola (Hexapoda) in Mazandaran Province." MSc thesis, Sari Agricaltural Science and Natural Resources University.

[7] Yoosefi Lafooraki, E., and Shayanmehr, M. 2014. "A Survey on Entomobryomorpha (Collembola, Hexapoda) Fauna in Northern Iran with an Identification Key." Iranian Journal of Animal Biosystematics 10 (2): 101-17.

[8] Yoosefi Lafooraki, E., and Shayanmehr, M. 2015. "New Addition to Fauna and a Checklist of the Order Poduromorpha (Collembola) of Mazandaran Province, Iran.” Far Eastern Entomologist 298: 1-10.

[9] Yoosefi Lafooraki, E., and Shayanmehr, M. 2015. "Identification Key and Introduction of New Species of Order Symphypleona (Collembola, Hexapoda) from Iran." Journal of Plant Protection 29 (2): 259-64. (in Persian)

[10] Yoosefi Lafooraki, E., and Shayanmehr, M. 2015. "First Record of the Genus Schoettella and Three New Records of the Family Hypogastruridae (Collembola, Hexapoda) for Fauna of Iran with an Identification Key for Mazandaran Province." Taxonomy and Biosystematics 7 (23): 1-12.

[11] Balvasi, A., Chelav, H. S., Khashaveh, A., and Shayanmehr, M. 2015. "New Report of Collembola 
(Hexapoda: Enthognatha) from North of Iran (Larijan Region)." International Journal of Agriculture and Biosciences 5 (1): 24-8.

[12] Mehrafrooz Mayvan, M., Shayanmehr, M., and Scheu, S. 2015. "Depth Distribution and Inter-annual Fluctuations in Density and Diversity of Collembola in an Iranian Hyrcanian Forest.” Soil Organisms 87 (3): 239-47.

[13] Mehrafrooz Mayvan, M., Shayanmehr, M., Smolis, A., and Skarżyński, D. 2015. "Persanura hyrcanica, a New Genus and Species of Neanurinae (Collembola: Neanuridae) from Iran, with a Key to Genera of the Tribe Neanurini." Zootaxa 3918 (4): 552-8.

[14] Potapov, M., Kahrarian, M., Deharveng, L., and Shayanmehr, M. 2015. "Taxonomy of the Proisotoma Complex. V. Sexually Dimorphic Ephemerotoma Gen. Nov. (Collembola: Isotomidae)." Zootaxa 4052 (3): 345-58.

[15] Smolis, A., Shayanmehr, M., Kuznetsova, N., and Yoosefi Lafooraki, E. 2017. "Three New Remarkable Species of the Genus Endonura Cassagnau, 1979 from the Middle East and Central Asia (Collembola, Neanuridae, Neanurinae, Neanurini)." Zoo Keys 673: 135-51.

[16] Smolis, A., Shayanmehr, M., and Yoosefi Lafooraki, E. 2018. "New Members of the Genera Neanura MacGillivray, 1893 and Deutonura Cassagnau, 1979 (Collembola: Neanuridae) from the Middle East." European Journal of Taxonomy 406: 1-16.

[17] Alijani-Ardeshir, M., Shayanmehr, M., and Amiri-Besheli, B. 2017. "Faunistic Survey of Collembola in Babol Regions, Mazandaran Province: Introduction New Species for Iranian Fauna." Plant Protection (Scientific Journal of Agriculture) 40 (2): 45-61. (in
Persian)

[18] Ghasemi-Cherati, M. 2017. "The Comparison Fauna, Diversity and Density of Collembola in an Agricultural Ecosystem versus a Forest Ecosystem." MSc thesis, Sari Agricultural Science and Natural Resources University.

[19] Kaprus', I., Shayanmehr, M., Kahrarian, M., and Yoosefi Lafooraki, E. 2017. "Three New Species of Onychiuridae Lubbock, 1871 (Collembola, Poduromorpha) from Iran." Zootaxa 4291 (2): 335-46.

[20] Yahyapour, E., Vafaei-Soushtari, R., and Shayanmehr, M. 2018. "Fauna Introduction and New Records of Collembola (Hexapoda) from Some Forests of Mazandaran (Iran).”

[21] Yahyapour, E., Vafaei-Soushtari, R., Shayanmehr, M., and Arbea, J. 2018. "A Survey on Entomobryomorpha (Collembola) Fauna in northern Iran.” Journal of Insect Biodiversity and Systematics 4 (4): 307-16.

[22] Yahyapour, E., Vafaei-Shoushtari, R., Shayanmehr, M., Goderio, N. N., and Bellini, B. C. 2018. "First Report of Genus Aethiopella Handschin, 1942 and Species Folsomia inoculata Stach, 1947 (Hexapoda: Collembola) from Iran."

[23] Mohammadi Nodeheki, L., Shayanmehr, M. \& Yazdanian, M. 2017. “A Survey on Hypogastruridae (Collembola: Poduromorpha) fauna in Babol, Babolsar and Amol, Mazandaran Province." 2th National Conference of New Achievement in Agronomy and Plant Breeding.

[24] Bakhshi, S., et al. 2014. "The First Record of the genus Allacma Börner and the Species Allacma fusca (Collembola: Sminthuridae) from Iran. Taxonomy and Biosystematics." 18: 13-18. 